\title{
Robust Fault Detection and Estimation in Nonlinear Systems with Unknown Constant Time-Delays
}

\author{
Wenxu Yan, ${ }^{1}$ Dezhi Xu, ${ }^{1}$ and Qikun Shen ${ }^{2}$ \\ ${ }^{1}$ School of Internet of Things Engineering, Jiangnan University, Wuxi 214122, China \\ ${ }^{2}$ College of Information Engineering, Yangzhou University, Yangzhou 225009, China \\ Correspondence should be addressed to Dezhi Xu; xudezhi@jiangnan.edu.cn
}

Received 23 March 2017; Accepted 29 May 2017; Published 7 August 2017

Academic Editor: Xinggang Yan

Copyright (C) 2017 Wenxu Yan et al. This is an open access article distributed under the Creative Commons Attribution License, which permits unrestricted use, distribution, and reproduction in any medium, provided the original work is properly cited.

\begin{abstract}
This paper studies the problem of fault detection and estimation in nonlinear time-delayed systems with unknown inputs, where the time-delays are supposed to be constant but unknown. A new fault detection filter, which can estimate online the time-delays, is first introduced. Then, a reference residual model is proposed to formulate the robust fault detection filter design problem as an $H_{\infty}$ model-matching problem. Furthermore, by a novel robust adaptive fault estimation algorithm, the classical assumption that the time derivative of the output error should be known is removed. In addition, applying a robust $H_{\infty}$ optimization control technique, sufficient conditions for the existence of the fault detection filter (FDF) are derived in terms of linear matrix inequality (LMI). Finally, simulation results are presented to illustrate the effectiveness of the proposed algorithm.
\end{abstract}

\section{Introduction}

Modern industrial control systems become more and more complex, with an increasing number of interconnected physical components, such as actuators and sensors [1]. In the system life, faults inevitably occur in these elements, which leads to the drop of systems performances, or even worse to system damage, with dramatic consequences on the environment. Consequently, in parallel with the development of high-performance technological systems, industrials express a growing demand for reliability, maintainability, and survivability [2]. Fault Tolerant Control (FTC) is an effective way of maintaining system performances under faulty conditions. FTC can be achieved either by a passive way or using an active method. Passive FTC uses feedback control laws that are robust with respect to an a priori fixed set of possible system faults $[3,4]$. On the other hand, active FTC uses a Fault Detection and Isolation (FDI) module that provides online fault information. Active FTC may consist of a fault accommodation scheme $[5,6]$, which is in fact an adaptive control with respect to the fault information, or may be a control system reconfiguration scheme, which consists of a switching control with a supervision layer that selects the most suitable control law for the identified faulty situation [7-10]. One difficulty is that FDI module and active FTC algorithm have to be designed jointly because of their mutual interactions.

In distributed or large-scale processes, the dynamic contains time-delays which may cause instability and degrade the system performances [11]. These time-delays may generally be considered as constant ones for given operating conditions. However these constant values are generally not known. The existence of such time-delays renders the control design problem much more difficult [12]. Increasing attention has recently been devoted to stability control and fault diagnosis of linear/nonlinear time-delayed systems, see, for example, [4-6, 11-16]. In [14], an observer-based fuzzy control scheme with adaptation to the time-delay was proposed. In [15], fault detection and identification for uncertain linear timedelay systems were investigated. In [16], by using $H_{\infty}$ control theory, a robust fault detection scheme was proposed for a class of discrete time-delay systems with parameter uncertainty. However, the sensitivity of the residual signals to the faults was not studied. In [17], the fault detection filter design problem for linear time-delay systems was studied by introducing an idealized reference residual model. Based 
on $[16,17]$, the robust fault detection filter (RFDF) design problem was studied in [18] for nonlinear time-delay systems with unknown inputs. In [19], $H_{\infty}$ fault detection filter design for linear discrete time systems with multiple time-delays was discussed. The fault detection filter design problem was investigated in [20] for LTI (Linear Time-Invariant) system with time-delays. In [21, 22], the fault estimation problem for linear systems with state time-delays was studied, and an adaptive fault detection observer was designed. Many observers that are proposed in the literature, as in [16-23], use explicitly the time-delay value that is supposed to be accessible. However, in practical applications, this time-delay is generally not available, which makes these techniques not applicable. Another drawback of many diagnosis techniques that are proposed in the literature is that they need the derivative of the output signal for the fault estimation algorithm. These derivatives are generally not directly measured and are difficult to compute in a noisy environment.

In this paper, we investigate the problem of fault detection and estimation of nonlinear time-delayed systems. Five main contributions are worth emphasis.

(1) Compared with some results (see [16-22], for instance), by online estimating the real value of state time-delay, the assumption that the time-delay must be a priori known is removed.

(2) An adaptive fault estimation algorithm is proposed where the classical assumption that the time derivative of the output errors has to be known (see [22], for instance) and is removed. Moreover, our scheme is robust to bounded disturbances.

(3) Differing from numerous FDI schemes in the literature, the bounds of the time derivatives of the faults have not been known in our proposed adaptive fault estimation algorithm.

(4) In this paper, in contrast with [18], not only the fault detection filter design problem is discussed, but also an adaptive fault estimation algorithm is proposed.

(5) Sufficient conditions for the existence of the adaptive fault observer are expressed using the Lyapunov stability theory.

The paper is organized as follows. The problem formulation is presented in Section 2. In Section 3, the main technical results of this paper are given, which include the choice of the reference residual model, the design of a robust fault detection observer/filter, and the fault estimation algorithm. Simulations are presented in Section 4. Finally, Section 5 draws the conclusion.

\section{Problem Formulation}

Consider the following class of nonlinear time-delay systems [18]:

$$
\begin{aligned}
\dot{x}(t)= & A x(t)+A_{d} x(t-h)+B u(t) \\
& +G g(x(t), x(t-h))+B_{f} f(t)+B_{d} d(t),
\end{aligned}
$$

$$
\begin{aligned}
& y(t)=C x(t)+D u(t)+D_{f} f(t)+D_{d} d(t), \\
& x(t)=0, \quad t \in[-\bar{h}, 0]
\end{aligned}
$$

where $x(t) \in R^{n}$ is the unknown state vector, $u(t) \in R^{p}$ is the control input vector, $y(t) \in R^{q}$ is the measured output, $d(t) \in$ $R^{m}$ that belongs to $L_{2}^{m}[0,+\infty]$ is an exogenous disturbance input vector that regroups all the model uncertainties, $f(t) \epsilon$ $R^{l}$ is the fault vector to be detected, $h \geq 0$ is an unknown but constant time-delay that satisfies $h \leq \bar{h}$, where $\bar{h}$ is a known constant, $g(\cdot, \cdot): R^{n} \times R^{n} \rightarrow R^{n_{g}}$ is a known nonlinear function, and $A, A_{d}, B, B_{f}, B_{d}, C, D, D_{f}, D_{d}$, $G$ are all known matrices with appropriate dimensions.

Throughout this paper, the following assumptions are made.

Assumption 1. The pair $(C, A)$ is detectable.

Assumption 2 (Lipschitz condition). It is supposed that $g(0,0)=0$ and $\left\|g\left(x_{1}, x_{2}\right)-g\left(y_{1}, y_{2}\right)\right\| \leq\left\|\rho_{1}\left(x_{1}-y_{1}\right)\right\|+$ $\left\|\rho_{2}\left(x_{2}-y_{2}\right)\right\|, \forall x_{1}, x_{2}, y_{1}, y_{2} \in R^{n}$, where $\rho_{1}>0 \in R, \rho_{2}>$ $0 \in R$, are known constant.

To detect the fault, the following so-called fault detection filter (FDF) is proposed:

$$
\begin{aligned}
\dot{\hat{x}}(t)= & A \widehat{x}(t)+A_{d} \widehat{x}(t-\widehat{h})+B u(t) \\
& +G g(\widehat{x}(t), \widehat{x}(t-\widehat{h}))+H[y(t)-\widehat{y}(t)], \\
\widehat{y}(t)= & C \widehat{x}(t)+D u(t), \\
r(t)= & V[y(t)-\widehat{y}(t)],
\end{aligned}
$$

where $\widehat{x}(t) \in R^{n}$ and $\widehat{y}(t) \in R^{q}$ denote the state and the output of the filter, respectively; $\widehat{h}$ is the estimation of the unknown constant time-delay $h ; r(t)$ is the so-called residual signal. The observer gain matrix $H$ and the residual weighting matrix $V$ will be defined later.

For the above observer design, the following assumption is made.

Assumption 3. There exists a known constant $M>0 \in R$ such that $\|x(t)-\widehat{x}(t)\| \leq M$.

Remark 4. Assumption 3 is not restrictive. In fact, this is a common assumption in the literature concerning filter/observer design. In practical application, it may be difficult to know accurately the upper bound of the observer error. Therefore, $M$ can be chosen large in practical applications. It must be pointed out that $M$ is only needed for stability analysis of the closed-loop system. Choosing a large $M$ does not degrade the performance of the closed-loop system.

Define

$$
e=x(t)-\widehat{x}(t) \text {. }
$$


The error dynamics can be computed as

$$
\begin{aligned}
\dot{e}(t)= & (A-H C) e(t)+A_{d} \widehat{x}(t-\widehat{h})-A_{d} x(t-h) \\
& +\left(B_{f}-H D_{f}\right) f(t)+\left(B_{d}-H D_{d}\right) d(t)+G \Psi \\
= & (A-H C) e(t)+A_{d} \widehat{x}(t-\widehat{h})-A_{d} \widehat{x}(t-h) \\
& +A_{d} \widehat{x}(t-h)-A_{d} x(t-h) \\
& +\left(B_{f}-H D_{f}\right) f(t)+\left(B_{d}-H D_{d}\right) d(t)+G \Psi \\
= & (A-H C) e(t)+A_{d} e(t-h)+A_{d} \widehat{x}(t-\widehat{h}) \\
& -A_{d} \widehat{x}(t-h)+\left(B_{f}-H D_{f}\right) f(t) \\
& +\left(B_{d}-H D_{d}\right) d(t)+G \Psi,
\end{aligned}
$$

where $\Psi=g(x(t), x(t-h))-g(\widehat{x}(t), \widehat{x}(t-\widehat{h}))$.

From Newton-Leibniz formula, we have

$$
\widehat{x}(t-\widehat{h})-\widehat{x}(t-h)=\int_{-h}^{-\widehat{h}} \dot{\hat{x}}(t+s) d s .
$$

Thus, error dynamics (4) can further be described as

$$
\begin{aligned}
\dot{e}(t)= & (A-H C) e(t)+A_{d} e(t-h) \\
& +A_{d} \int_{-h}^{-\hat{h}} \dot{\hat{x}}(t+s) d s+\left(B_{f}-H D_{f}\right) f(t) \\
& +\left(B_{d}-H D_{d}\right) d(t)+G \Psi, \\
r(t)= & V C e(t)+V D_{f} f(t)+V D_{d} d(t) .
\end{aligned}
$$

From (6), it is seen that the dynamics of the residual signal depends not only on fault $f(t)$ and uncertainty $d(t)$, but also on the nonlinear part $\Psi$. Motivated by the method presented in [18], we propose using a reference residual model that describes the desired behavior of the residual vector $r(t)$, to formulate the RFDF design problem as an $H_{\infty}$ modelmatching problem. In other words, the objectives are finding an idealized reference residual and minimizing the distance between the generated residual and the idealized reference residual. In the idealized case, the observed state $\widehat{x}(t)$ and the time-delayed state $\widehat{x}(t-\widehat{h})$ should be equal, respectively, to $x(t)$ and $x(t-h)$; thus we have $x(t)-\widehat{x}(t)=0$ and $x(t-h)-\widehat{x}(t-\widehat{h})=0$. Consequently, $\Psi=g(x(t), x(t-$ $h))-g(\widehat{x}(t), \widehat{x}(t-\widehat{h}))=0$. Therefore, according to (6), and assuming $\Psi=0$, the corresponding reference residual error model is given by

$$
\begin{aligned}
& \dot{e}_{f}(t)=(A-\bar{H} C) e_{f}(t)+A_{d} e_{f}(t-h) \\
&+\left(B_{f}-\bar{H} D_{f}\right) f(t)+\left(B_{d}-\bar{H} D_{d}\right) d(t), \\
& r_{f}(t)=\bar{V} C e_{f}(t)+\bar{V} D_{f} f(t)+\bar{V} D_{d} d(t), \\
& e_{f}(t)=0(t \leq 0),
\end{aligned}
$$

where $e_{f}(t) \in R^{n}$ is the reference model error state vector, $r_{f}(t)$ is the reference model residual signal, and $\bar{H}$ and $\bar{V}$ are the parameters of the reference residual model to be designed.

Thus the overall system can be described by

$$
\begin{aligned}
\dot{\eta}(t)= & \bar{A} \eta(t)+\bar{A}_{d} \eta(t-h)+\overline{\bar{A}}_{d} \int_{-h}^{-\widehat{h}} \dot{\hat{x}}(t+s) d s \\
& +\bar{B} w(t)+\bar{G} \Psi, \\
r_{e}(t)= & r(t)-r_{f}(t)=\bar{C} \eta(t)+\bar{D} w(t),
\end{aligned}
$$

where

$$
\begin{aligned}
& \eta(t)=\left[\begin{array}{c}
e(t) \\
e_{f}(t)
\end{array}\right], \\
& w(t)=\left[\begin{array}{l}
f \\
d
\end{array}\right], \\
& \bar{A}=\left[\begin{array}{cc}
A-H C & 0 \\
0 & A-\bar{H} C
\end{array}\right], \\
& \bar{A}_{d}=\left[\begin{array}{cc}
A_{d} & 0 \\
0 & A_{d}
\end{array}\right] \text {, } \\
& \overline{\bar{A}}_{d}=\left[\begin{array}{c}
A_{d} \\
0
\end{array}\right] \text {, } \\
& \bar{G}=\left[\begin{array}{l}
G \\
0
\end{array}\right], \\
& \bar{B}=\left[\begin{array}{ll}
B_{f}-H D_{f} & B_{d}-H D_{d} \\
B_{f}-\bar{H} D_{f} & B_{d}-\bar{H} D_{d}
\end{array}\right], \\
& \bar{C}=\left[\begin{array}{ll}
V C & -\bar{V} C
\end{array}\right], \\
& \bar{D}=\left[\begin{array}{lll}
V D_{f}-\bar{V} D_{f} & V D_{d}-\bar{V} D_{d}
\end{array}\right] .
\end{aligned}
$$

From (8), the design of the robust fault detection filter, which is the main objective of this work, may be formulated as an $H_{\infty}$ model-matching design problem. Applying a robust $H_{\infty}$ optimization control technique, for all exogenous disturbance inputs and nonlinear parts, the generated residual $r(t)$ will be designed as closely as possible to the reference model residual $r_{f}(t)$, independently of the unknown time-delay $h$. Thus, the problem of designing an observer-based RFDF can be described as designing the observer gain matrix $H$ and the residual weighting matrix $V$ such that

(i) systems (8) are robustly asymptotically stable;

(ii) under zero initial condition, for given constant $\gamma>0$ and any nonzero $w(t) \in L_{2}[0, \infty)$, systems (8) satisfy the following inequality:

$$
\left\|r_{e}(t)\right\|_{2}<\gamma\|w(t)\|_{2} \text {. }
$$

After designing the RFDF, the remaining important task is to decide, from the generated residual, if an alarm has to be 
generated. One of the widely adopted approaches is to choose a so-called threshold $J_{\text {th }}>0$ and, based on this, use the following logical relationship for fault detection:

$$
\begin{aligned}
& \|r(t)\|_{2, \tau}>J_{\text {th }} \Longrightarrow \text { a fault has occured } \Longrightarrow \text { alarm } \\
& \|r(t)\|_{2, \tau}<J_{\text {th }} \Longrightarrow \text { no fault has occured, }
\end{aligned}
$$

where $\|r(t)\|_{2, \tau}=\left[\int_{t_{1}}^{t_{2}} r(t)^{T} r(t) d t\right]^{1 / 2}, \tau=t_{1}-t_{2}, t \in\left[t_{1}, t_{2}\right]$.

The threshold $J_{\text {th }}>0$ can be chosen as $J_{\text {th }}=\bar{f}+\bar{d}$, where $\bar{f}>0 \in R$ and $\bar{d}>0 \in R$ denote, respectively, the upper bound of the norm of fault $f$ and exogenous disturbance $d$, that is, $\|f\|_{2} \leq \bar{f},\|d\|_{2} \leq \bar{d}$.

Remark 5. There are many results on fault detection observer/filter design for time-delayed systems in the literature. In general, the observer, as the one in $[16,18]$, is designed in the following form:

$$
\begin{aligned}
\dot{\hat{x}}(t)= & A \hat{x}(t)+A_{d} \widehat{x}(t-h)+B u(t) \\
& +G g(\widehat{x}(t), \widehat{x}(t-h)) \\
& +G g(\widehat{x}(t), \widehat{x}(t-h))+H[y(t)-\widehat{y}(t)], \\
\widehat{y}(t)= & C \widehat{x}(t)+D u(t), \\
r(t)= & V[y(t)-\widehat{y}(t)] .
\end{aligned}
$$

However, just as said in [14], the shortcoming of the abovementioned observer is that the constant state time-delay $h$ must be exactly known. If the time-delay $h$ is unknown, the above observer will not work in practical applications.

\section{Main Results and Proof}

As mentioned in [24, 25], the design of a RFDF for the nonlinear time-delay system (1) can be formulated as an $H_{\infty}$ model-matching problem. In this section, we first design the reference residual model and then express the RFDF design problem under an LMI formulation.

3.1. Choice of the Reference Residual Model. As pointed out in [18], the selection of a suitable reference residual model is one of the key steps to design an RFDF for nonlinear timedelay systems. If the reference residual model is not selected suitably, miss alarms or false alarms may occur. In order to select a suitable reference residual model, we consider the following performance index $J_{f}$ :

$$
J_{f}=\left\|T_{r_{f} d}(s)\right\|_{\infty}-\left\|T_{r_{f} f}(s)\right\|_{\infty},
$$

where $T_{r_{f} d}(s)$ and $T_{r_{f} f}(s)$ are the transfer functions from $f$ and $d$ to the reference model residual $r_{f}$.

Notice that if $J_{f} \rightarrow \min \left\{J_{f}\right\}$, then one has

$$
\begin{aligned}
\left\|T_{r_{f} d}(s)\right\|_{\infty} & \longrightarrow \max \left\{T_{r_{f} d}(s)\right\}, \\
\left\|T_{r_{f} f}(s)\right\|_{\infty} & \longrightarrow \min \left\{T_{r_{f} f}(s)\right\} .
\end{aligned}
$$

Therefore, according to the performance index $J_{f}$ (13), the reference residual model can be designed, which takes into account not only the robustness of the reference model residual against disturbance but also the sensitivity to faults.

For the sake of simplicity, we assume that $l=m$.

Consider the following transfer function:

$$
T=M T_{r_{f} \vartheta} N=M\left[T_{r_{f} f} T_{r_{f} d}\right] N,
$$

where the matrices $M \in R^{q \times q}, N \in R^{2 l \times l}$ select the appropriate input/output channels or channels combinations and $T$ satisfies the following equalities:

$$
T\left\{\begin{array}{l}
\dot{e}_{f}(t)=(A-\bar{H} C) e_{f}(t)+A_{d} e_{f}(t-h)+B_{1} \vartheta(t) \\
r_{f}(t)=M \bar{V} C e_{f}(t)+M D_{1} N \vartheta(t)
\end{array}\right.
$$

where $B_{1}=\left[\begin{array}{ll}B_{f}-\bar{H} D_{f} & B_{d}-\bar{H} D_{d}\end{array}\right], D_{1}=\left[\begin{array}{ll}D_{f} & D_{d}\end{array}\right]$, and $\vartheta(t)=[d-f]$.

Choosing $M=I_{q \times q}, N=\left[\begin{array}{ll}-I_{l \times l} & I_{l \times l}\end{array}\right]^{T}$, and giving $\alpha>0$, it follows that

$$
\begin{aligned}
& \|T\|_{\infty}=\left\|T_{r_{f} d}-T_{r_{f} f}\right\|_{\infty}>\left\|T_{r_{f} d}\right\|_{\infty}-\left\|T_{r_{f} f}\right\|_{\infty}, \\
& \|T\|_{\infty}<\alpha \Longrightarrow\left\|T_{r_{f} d}\right\|_{\infty}-\left\|T_{r_{f} f}\right\|_{\infty}<\alpha .
\end{aligned}
$$

Then the reference residual model can be designed by solving the following optimization problem:

$$
\begin{array}{ll}
\min & \alpha \\
\text { s.t. } & (13),(17) \text { hold. }
\end{array}
$$

The following theorem provides a sufficient condition to ensure that, for a given $\alpha>0$, the reference RFDF satisfies (16).

Theorem 6. Given $\alpha>0$ and the reference residual model (7), if there exist symmetric matrices $P=P^{T}>0, Q=Q^{T}>0$, $Z>0$, and $Y$ such that

$$
\Xi=\left[\begin{array}{ccc}
A^{T} P+P A-C^{T} Y^{T}-Y C+C^{T} Z C+Q & P\left(B_{d}-B_{f}\right)+Y\left(B_{f}-B_{d}\right)+C^{T} Z\left(D_{d}-D_{f}\right) & P A_{d} \\
* & \left(D_{d}-D_{f}\right)^{T} Z\left(D_{d}-D_{f}\right)-\alpha^{2} I & 0 \\
* & * & -Q
\end{array}\right]<0
$$

holds and applying the time-delay adaptive law

$$
\dot{\hat{h}}=-\frac{1}{\eta} M\left(\left\|P A_{d}\right\|+\left\|P \bar{G} \rho_{2}\right\|\right) \alpha(t), \quad \widehat{h}(0)>\bar{h}
$$

then system (7), subject to Assumptions 1-3, is stable and satisfies $\left\|T_{r_{f} d}\right\|_{\infty}-\left\|T_{r_{f} f}\right\|_{\infty}<\alpha$, where $\alpha(t)=\|\dot{\hat{x}}(t)\|, \eta>0$, $\widehat{h}(t) \geq \bar{h}, \bar{H}=P^{-1} Y$, and $\bar{V}=Z^{1 / 2}$. 
Proof. Consider the following Lyapunov-Krasovskii function:

$$
\begin{aligned}
V_{1}(t)= & e_{f}^{T}(t) P e_{f}(t)+\int_{t-h}^{t} e_{f}^{T}(\tau) Q e_{f}(\tau) d \tau \\
& +2 M\left\|P A_{d}\right\| \int_{-\widehat{h}}^{-h} d \theta \int_{\theta}^{0} \alpha(t+s) d s \\
& +\frac{\eta}{2}(\widehat{h}-h)^{2} .
\end{aligned}
$$

Differentiating $V_{1}$ with respect to time $t$, one has

$$
\begin{aligned}
\dot{V}_{1} \leq & e_{f}^{T}(t)\left(P(A-\bar{H} C)+(A-\bar{H} C)^{T} P\right) e_{f}(t) \\
& +e_{f}^{T}(t) P A_{d} A_{d}^{T} P e_{f}(t)+e_{f}^{T}(t-h) Q e_{f}(t-h) \\
& +2 e_{f}^{T}(t) P A_{d} \int_{-h}^{-\widehat{h}} \dot{\hat{x}}(t+s) d s+e_{f}^{T}(t) Q e_{f}(t) \\
& -e_{f}^{T}(t-h) Q e_{f}(t-h)+\eta(\widehat{h}-h) \dot{\hat{h}} \\
& -2 M\left(\left\|P A_{d}\right\|+\left\|P \bar{G} \rho_{2}\right\|\right)[(\widehat{h}-h) \alpha(t) \\
& \left.-\int_{-\widehat{h}}^{-h} \alpha(t+\theta) d \theta+\dot{\hat{h}} \int_{-\widehat{h}}^{0} \alpha(t+s) d s\right] .
\end{aligned}
$$

Since $\dot{\hat{h}}=-(1 / \eta) M\left\|P A_{d}\right\| \alpha(t) \leq 0$, one has $\dot{\hat{h}} \int_{-\hat{h}}^{0} \alpha(t+$ $s) d s \leq 0$. Under idealized condition, using the FDF (2), the observer error is asymptotically converging to zero; that is, $x(t)-\widehat{x}(t) \rightarrow 0, x(t-h)-\widehat{x}(t-\widehat{h}) \rightarrow 0$.

Then, one has

$$
\begin{aligned}
\dot{V}_{1} & \leq e_{f}^{T}(t) \\
& \cdot\left(P(A-\bar{H} C)+(A-\bar{H} C)^{T} P+P A_{d} A_{d}^{T} P+Q\right) \\
& \cdot e_{f}(t) .
\end{aligned}
$$

Define the following performance index $J_{1}$ :

$$
\begin{aligned}
J_{1}= & \int_{0}^{\infty} r_{f}^{T}(t) r_{f}(t) d t-\alpha^{2} \int_{0}^{\infty} \vartheta^{T}(t) \vartheta(t) d t \\
= & \int_{0}^{\infty}\left[r_{f}^{T}(t) r_{f}(t)-\alpha^{2} \vartheta^{T}(t) \vartheta(t)+\dot{V}_{1}(t)\right] d t \\
& -\left.V_{1}(t)\right|_{t}+\left.V_{1}(t)\right|_{t=0} .
\end{aligned}
$$

Then, we have

$$
J_{1}<\int_{0}^{\infty}\left[r_{f}^{T}(t) r_{f}(t)-\alpha^{2} \vartheta^{T}(t) \vartheta(t)+\dot{V}_{1}(t)\right] d t
$$

Let $Y=P \bar{H}, Z=\bar{V}^{T} \bar{V}, M=I_{q \times q}$, and $N=\left[\begin{array}{ll}-I_{l \times l} & I_{l \times l}\end{array}\right]^{T}$; then

$$
\begin{aligned}
r_{f}^{T} r_{f}= & \left(M \bar{V} C e_{f}+M D_{1} N \vartheta\right)^{T}\left(M \bar{V} C e_{f}+M D_{1} N \vartheta\right) \\
= & \left(\bar{V} C e_{f}+D_{1}\left[\begin{array}{ll}
-\vartheta & \vartheta
\end{array}\right]^{T}\left(\bar{V} C e_{f}+D_{1}\left[\begin{array}{ll}
-\vartheta & \vartheta
\end{array}\right]^{T}\right)\right. \\
= & \left(e_{f}^{T} C^{T} \bar{V}^{T}+\left[\begin{array}{ll}
-\vartheta & \vartheta
\end{array}\right] D_{1}^{T}\right)\left(\bar{V} C e_{f}+D_{1}\left[\begin{array}{ll}
-\vartheta & \vartheta
\end{array}\right]^{T}\right) \\
= & e_{f}^{T} C^{T} \bar{V}^{T} \bar{V} C e_{f}-e_{f}^{T} C^{T} \bar{V}^{T} D_{f} \vartheta^{T}+e_{f}^{T} C^{T} \bar{V}^{T} D_{d} \vartheta^{T} \\
& -\vartheta D_{f}^{T} \bar{V} C e_{f}+\vartheta D_{f}^{T} D_{f} \vartheta^{T}-\vartheta D_{f}^{T} D_{d} \vartheta^{T} \\
& +\vartheta D_{d}^{T} \bar{V} C e_{f}-\vartheta D_{d}^{T} D_{f} \vartheta^{T}+\vartheta D_{d}^{T} D_{d} \vartheta^{T} .
\end{aligned}
$$

Therefore,

$$
J_{1}<\int_{0}^{\infty} \Delta d t
$$

where

$$
\begin{aligned}
\Delta & =e_{f}^{T} C^{T} \bar{V}^{T} \bar{V} C e_{f}-e_{f}^{T} C^{T} \bar{V}^{T} D_{f} \vartheta^{T}+e_{f}^{T} C^{T} \bar{V}^{T} D_{d} \vartheta^{T} \\
& -\vartheta D_{f}^{T} \bar{V} C e_{f}+\vartheta D_{f}^{T} D_{f} \vartheta^{T}-\vartheta D_{f}^{T} D_{d} \vartheta^{T} \\
& +\vartheta D_{d}^{T} \bar{V} C e_{f}-\vartheta D_{d}^{T} D_{f} \vartheta^{T}+\vartheta D_{d}^{T} D_{d} \vartheta^{T}+e_{f}^{T}(t) \\
& \cdot\left(P(A-\bar{H} C)+(A-\bar{H} C)^{T} P+P A_{d} A_{d}^{T} P+Q\right) \\
& \cdot e_{f}(t)+-\alpha^{2} \vartheta^{T}(t) \vartheta(t)
\end{aligned}
$$

which may be rewritten as

$$
J_{1}<\int_{0}^{\infty}\left[\begin{array}{c}
r_{f}(t) \\
\vartheta(t)
\end{array}\right]^{T} \Xi\left[\begin{array}{c}
r_{f}(t) \\
\vartheta(t)
\end{array}\right] d t .
$$

Since $\Xi<0$, we have $J_{1}<0$ and $\left\|T_{r_{f} d}(s)-T_{r_{f} f}(s)\right\|_{\infty}<\alpha$. And since $\left\|T_{r_{f} d}\right\|_{\infty}-\left\|T_{r_{f} f}\right\|_{\infty} \leq\left\|T_{r_{f} d}-T_{r_{f} f}\right\|_{\infty}$, we get $\left\|T_{r_{f} d}\right\|_{\infty}-$ $\left\|T_{r_{f} f}\right\|_{\infty}<\alpha$.

If $P(A-\bar{H} C)+(A-\bar{H} C)^{T} P+P A_{d} A_{d}^{T} P+Q<0$ holds, that is,

$$
\left[\begin{array}{cc}
A^{T} P+P A-Y C-C^{T} Y^{T}+Q & P A_{d} \\
A_{d}^{T} P & -Q
\end{array}\right]<0
$$

then, $\dot{V}_{1}<0$ under the condition $\vartheta=0$. If (19) holds, then (30) holds. Thus, system (7) is asymptotically stable.

The proof is completed.

3.2. Design of Robust Fault Detection Filter. In this section, we propose a theorem that gives a sufficient condition to guarantee that the RFDF system is stable and has a prescribed $H_{\infty}$ performance, independently of the time-delay. Before presenting the theorem, the following lemmas are introduced.

Lemma 7. Given constant matrices $\chi_{1}=\chi_{1}^{T}, \chi_{2}=\chi_{2}^{T}>0$, and $\chi_{3}$, then $\chi_{1}+\chi_{3}^{T} \chi_{2}^{-1} \chi_{3}<0$, if and only if 


$$
\begin{gathered}
{\left[\begin{array}{cc}
\chi_{1} & \chi_{3}^{T} \\
\chi_{3} & -\chi_{2}
\end{array}\right]<0} \\
\text { or equivalently }\left[\begin{array}{cc}
-\chi_{2} & \chi_{3} \\
\chi_{3}^{T} & -\chi_{1}
\end{array}\right]<0 .
\end{gathered}
$$

Lemma 8. Let $A$ and $B$ be real matrices of appropriate dimensions. For any scalar $\varepsilon>0$ and vectors $x, y \in R^{n}$, the following inequality holds:

$$
\left[\begin{array}{ccccc}
\Omega_{1} & 0 & P_{1} A_{d} & 0 & P_{1} B_{f}-Y D_{f} \\
* & \Omega_{2} & 0 & P_{2} A_{d} & P_{2}\left(B_{f}-\bar{H} D_{f}\right) \\
* & * & 2 \varepsilon \rho_{2}^{T} \rho_{2}-Q_{1} & 0 & 0 \\
* & * & * & Q_{2} & 0 \\
* & * & * & * & -\gamma^{2} I \\
* & * & * & * & * \\
* & * & * & * & * \\
* & * & * & * & *
\end{array}\right.
$$

holds and applying the time-delay adaptive law

$$
\dot{\hat{h}}=-\frac{1}{\eta} M\left(\left\|P \overline{\bar{A}}_{d}\right\|+\left\|P \bar{G} \rho_{2}\right\|\right) \alpha(t), \quad \widehat{h}(0)>h
$$

then system (8), subject to Assumptions 1-3, is stable and satisfies $\|z\|_{2}<\gamma\|w\|_{2}$, where $\widehat{h}(t) \geq \bar{h}, \Omega_{1}=P_{1} A+A^{T} P_{1}-$ $Y C-C^{T} Y^{T}+2 \varepsilon \rho_{1}^{T} \rho_{1}+Q_{1}, \Omega_{2}=P_{2} A+A^{T} P_{2}-P_{2} \bar{H} C-C^{T} \bar{H}^{T} P_{2}+$ $Q_{2}, \eta>0, \bar{H}=P^{-1} Y, \alpha(t)=\|\dot{\hat{x}}(t)\|$, and $Q-I>0 ; I$ denotes the identity matrix with appropriate dimensions.

Proof. Define the following Lyapunov-Krasovskii function:

$$
V_{2}=V_{1}^{\prime}+V_{2}^{\prime}+V_{3}^{\prime}+V_{4}^{\prime}
$$

with

$$
\begin{aligned}
& V_{1}^{\prime}=\eta^{T}(t) P \eta(t), \\
& V_{2}^{\prime}=\int_{t-h}^{t} \eta^{T}(s) Q \eta(s) d s, \\
& V_{3}^{\prime}=\frac{\sigma}{2}(\widehat{h}-h)^{2}, \\
& V_{4}^{\prime}=2 M\left(\left\|P \overline{\bar{A}}_{d}\right\|+\left\|P \bar{G} \rho_{2}\right\|\right) \int_{-\widehat{h}}^{-h} d \theta \int_{\theta}^{0} \alpha(t+s) d s,
\end{aligned}
$$

where $P=\left[\begin{array}{cc}P_{1} & 0 \\ 0 & P_{2}\end{array}\right], Q=\left[\begin{array}{cc}Q_{1} & 0 \\ 0 & Q_{2}\end{array}\right]$

$$
2 x^{T} A B y \leq \varepsilon^{-1} x^{T} A A^{T} x+\varepsilon y^{T} B^{T} B y .
$$

Recall the nonlinear time-delay system (8).

Theorem 9. For a given positive constant $\gamma>0$, if there exist a scalar $\varepsilon>0\left(2 \varepsilon \rho_{2}^{T} \rho_{2}<Q_{1}\right)$ and matrices $P_{1}=P_{1}^{T}>0$, $P_{2}=P_{2}^{T}>0, Q_{1}=Q_{1}^{T}>0, Q_{2}=Q_{2}^{T}>0, Y$, and $V$ such that the following LMI:

$$
\left.\begin{array}{ccc}
P_{1} B_{d}-Y D_{d} & C^{T} V^{T} & P_{1} G \\
P_{2}\left(B_{d}-\bar{H} D_{d}\right) & -C^{T} \bar{V}^{T} & 0 \\
0 & 0 & 0 \\
0 & 0 & 0 \\
0 & D_{f}^{T}\left(V^{T}-\bar{V}^{T}\right) & 0 \\
-\gamma^{2} I & D_{d}^{T}\left(V^{T}-\bar{V}^{T}\right) & 0 \\
* & -I & 0 \\
* & * & -\varepsilon I
\end{array}\right]
$$

Differentiating $V$ with respect to time $t$, one has

$$
\begin{aligned}
& \dot{V}_{1}^{\prime}(t)=2 \eta^{T}(t) P\left[\bar{A} \eta(t)+\bar{A}_{d} \eta(t-h)+\bar{G} \Psi+\bar{G} \Delta\right. \\
& +\bar{B} w(t)]+2 \eta^{T}(t) P \overline{\bar{A}}_{d} \int_{-\widehat{h}}^{-h} \dot{\hat{x}}(t+s) d s, \\
& \dot{V}_{2}^{\prime}(t)=\eta^{T}(t) Q \eta(t)-\eta^{T}(t-h) Q \eta(t-h), \\
& \dot{V}_{3}^{\prime}(t)=\sigma(\widehat{h}-h) \dot{\hat{h}}, \\
& \dot{V}_{4}^{\prime}(t)=2 M\left(\left\|P \overline{\bar{A}}_{d}\right\|+\left\|P \bar{G} \rho_{2}\right\|\right) \\
& \left.\quad \cdot\left\{\int_{-\widehat{h}}^{-h}[\alpha(t)-\alpha(t+\theta)] d \theta+\dot{\widehat{h}}\right]_{-\widehat{h}}^{0} \alpha(t+s) d s\right\} \\
& \quad=2 M\left(\left\|P \overline{\bar{A}}_{d}\right\|+\left\|P \bar{G} \rho_{2}\right\|\right)\left[\int_{-\widehat{h}}^{-h} \alpha(t) d \theta\right. \\
& \left.\quad+\dot{\hat{h}} \int_{-\widehat{h}}^{0} \alpha(t+s) d s\right] \cdot \\
& \left.\quad-\int_{-h}^{-\widehat{h}} \alpha(t+\theta) d \theta+\hat{\widehat{h}} \int_{-\widehat{h}}^{0} \alpha(t+s) d s\right] \\
& \quad+-2 M\left(\left\|P \overline{\bar{A}}_{d}\right\|+\left\|P \bar{G} \rho_{2}\right\|\right)
\end{aligned}
$$


From Assumption 2, it yields

$$
\begin{aligned}
\|\Psi\| \leq & \left\|\rho_{1}(x(t)-\widehat{x}(t))\right\| \\
& +\left\|\rho_{2}(x(t-h)-\widehat{x}(t-\widehat{h}))\right\| \\
\leq & \left\|\rho_{1} e(t)\right\|+\left\|\rho_{2}(\widehat{x}(t-h)-\widehat{x}(t-\widehat{h}))\right\| \\
= & \left\|\rho_{1} e(t)\right\|+\left\|\rho_{2} \int_{-\widehat{h}}^{-h} \dot{\hat{x}}(t+s) d s\right\| .
\end{aligned}
$$

From Lemma 8, one has

$$
\begin{aligned}
2 \eta^{T}(t) P \bar{G} \Psi \leq 2\left\|\eta^{T}(t)\right\| \cdot\|P \bar{G}\| \cdot\|\Psi\| \\
\leq 2\|\eta(t)\| \cdot\|P \bar{G}\| \\
\cdot\left(\left\|\rho_{1} e(t)\right\|+\left\|\rho_{2} \int_{-\widehat{h}}^{-h} \dot{\hat{x}}(t+s) d s\right\|\right) \\
\leq 2\|\eta(t)\| \cdot\|P \bar{G}\| \\
\cdot\left(\left\|\rho_{1} e(t)\right\|+\left\|\rho_{2}\right\| \int_{-\widehat{h}}^{-h}\|\dot{\hat{x}}(t+s)\| d s\right) \\
\leq 2\|\eta(t)\| \cdot\|P \bar{G}\| \\
\quad\left(\left\|\rho_{1} e(t)\right\|+\left\|\rho_{2}\right\| \int_{-\widehat{h}}^{-h} \alpha(t+s) d s\right) \\
\leq \varepsilon^{-1} \eta^{T}(t) P \bar{G} \bar{G}^{T} P \eta(t)+2 \varepsilon e^{T}(t) \rho_{1}^{T} \rho_{1} e(t) \\
+2\|\eta(t)\| \cdot\left\|P \bar{G} \rho_{2}\right\| \int_{-\widehat{h}}^{-h} \alpha(t+s) d s .
\end{aligned}
$$

Then

$$
\begin{aligned}
\dot{V}_{2} & \leq \varepsilon^{-1} \eta^{T}(t) P \bar{G} \bar{G}^{T} P \eta(t)+2 \varepsilon e^{T}(t) \rho_{1}^{T} \rho_{1} e(t) \\
& +2\|\eta(t)\| \cdot\|P \bar{G}\| \cdot\left\|\rho_{2}\right\| \int_{-\widehat{h}}^{-h} \alpha(t+s) d s+\eta^{T}(t) \\
& \cdot Q \eta(t)-\eta^{T}(t-h) Q \eta(t-h)+\sigma(\widehat{h}-h) \dot{\widehat{h}} \\
& -2 M\left\|P \overline{\bar{A}}_{d}\right\|\left[(\widehat{h}-h) \alpha(t)-\int_{-\widehat{h}}^{-h} \alpha(t+\theta) d \theta\right. \\
& \left.+\dot{\widehat{h}} \int_{-\widehat{h}}^{0} \alpha(t+s) d s\right] .
\end{aligned}
$$

Since $\dot{\hat{h}}=-(1 / \eta) M\left(\left\|P \overline{\bar{A}}_{d}\right\|+\left\|P \bar{G} \rho_{2}\right\|\right) \alpha(t)$, we have $\dot{\widehat{h}} \int_{-\bar{h}}^{0} \alpha(t+$ $s) d s \leq 0$. Because $\|x(t)-\widehat{x}(t)\|=\|e(t)\| \leq M$, and the above fault detection observer error is equal to zero under ideal condition, it holds that $\|\eta(t)\| \leq M$.
From Lemma 8, one has

$$
\begin{aligned}
\dot{V}_{2} \leq & 2 \eta^{T}(t) P\left[\bar{A} \eta(t)+\bar{A}_{d} \eta(t-h)+\bar{B} w(t)\right] \\
& +\varepsilon^{-1} \eta^{T}(t) P \bar{G} \bar{G}^{T} P \eta(t)+2 \varepsilon e^{T}(t) \rho_{1}^{T} \rho_{1} e(t) \\
& +\eta^{T}(t) Q \eta(t)-\eta^{T}(t-h) Q \eta(t-h) .
\end{aligned}
$$

And $e(t)=\left[\begin{array}{ll}I & 0\end{array}\right]\left[\begin{array}{c}e(t) \\ e_{f}(t)\end{array}\right]=\left[\begin{array}{ll}I & 0\end{array}\right] \eta(t), e(t-h)=$ $\left[\begin{array}{ll}I & 0\end{array}\right]\left[\begin{array}{c}e(t-h) \\ e_{f}(t-h)\end{array}\right]=\left[\begin{array}{ll}I & 0\end{array}\right] \eta(t-h)$. Thus, (41) can be rewritten as

$$
\begin{aligned}
& \dot{V}_{2} \leq 2 \eta^{T}(t) P\left[\bar{A} \eta(t)+\bar{A}_{d} \eta(t-h)+\bar{B} w(t)\right] \\
& +\varepsilon^{-1} \eta^{T}(t) P \bar{G} \bar{G}^{T} P \eta(t) \\
& +2 \varepsilon \eta^{T}(t)\left[\begin{array}{l}
I \\
0
\end{array}\right] \rho_{1}^{T} \rho_{1}\left[\begin{array}{l}
I \\
0
\end{array}\right]^{T} \eta(t)+\eta^{T}(t) Q \eta(t) \\
& -\eta^{T}(t-h) Q \eta(t-h) \\
& =\eta^{T}(t) P\left[\bar{A} \eta(t)+\bar{A}_{d} \eta(t-h)+\bar{B} w(t)\right] \\
& +\left[\bar{A} \eta(t)+\bar{A}_{d} \eta(t-h)+\bar{B} w(t)\right]^{T} P \eta(t) \\
& +\varepsilon^{-1} \eta^{T}(t) P \bar{G} \bar{G}^{T} P \eta(t) \\
& +2 \varepsilon \eta^{T}(t)\left[\begin{array}{cc}
\rho_{1}^{T} \rho_{1} & 0 \\
0 & 0
\end{array}\right] \eta(t)+\eta^{T}(t) Q \eta(t) \\
& -\eta^{T}(t-h) Q \eta(t-h) .
\end{aligned}
$$

Consider the following performance index:

$$
\begin{aligned}
& J_{2}= \int_{0}^{\infty} r_{e}^{T}(t) r_{e}(t) d t-\gamma^{2} \int_{0}^{\infty} w^{T}(t) w(t) d t \\
&= \int_{0}^{\infty}\left[r_{e}^{T}(t) r_{e}(t)-\gamma^{2} w^{T}(t) w(t)+\dot{V}_{2}(t)\right] d t \\
&-\left.V_{2}(t)\right|_{t}+\left.V_{2}(t)\right|_{t=0} \\
& \leq \int_{0}^{\infty}\left[\begin{array}{c}
\left.r_{e}^{T}(t) r_{e}(t)-\gamma^{2} w^{T}(t) w(t)+\dot{V}_{2}(t)\right] d t \\
\leq
\end{array}\right. \\
& \int_{0}^{\infty}\left[\begin{array}{c}
\eta(t) \\
\eta(t-h) \\
w(t)
\end{array}\right]\left[\begin{array}{c}
\eta(t) \\
\eta(t-h) \\
w(t)
\end{array}\right] d t,
\end{aligned}
$$


where

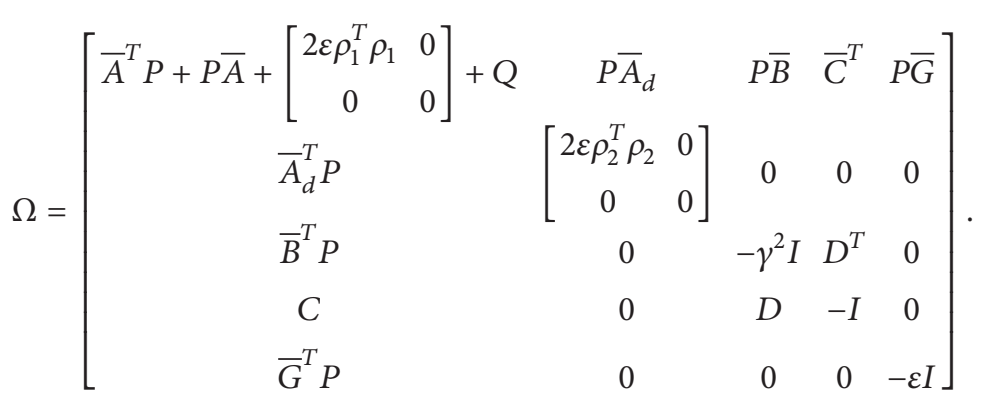

From (35), one has $\Omega<0$. Hence $J_{2}<0$ and $\|z\|_{2}<\gamma\|w\|_{2}$.

If $w(t)=0$, from the above analysis, one has

$$
J_{2} \leq \int_{0}^{\infty}\left[\begin{array}{c}
\eta(t) \\
\eta(t-h) \\
w(t)
\end{array}\right]^{T} \Omega^{\prime}\left[\begin{array}{c}
\eta(t) \\
\eta(t-h) \\
w(t)
\end{array}\right] d t
$$

where $\Omega^{\prime}=\left[\begin{array}{ccc}\bar{A}^{T} P+P \bar{A}+\varepsilon^{-1} P \bar{G} \bar{G}^{T} P+2 \varepsilon \rho_{1}^{T} \rho_{1}+\bar{C}^{T} \overline{\mathrm{C}}+\mathrm{Q} & P \bar{A}_{d} & 0 \\ \bar{A}_{d}^{T} P & -Q & 0 \\ 0 & 0 & -\gamma^{2} I\end{array}\right]$.

Obviously, $\Omega<0 \Rightarrow \Omega^{\prime}<0$. Furthermore, transferring $\Omega^{\prime}$, one has

$$
\left[\begin{array}{ccc}
\bar{A}^{T} P+P \bar{A}+2 \varepsilon \rho_{1}^{T} \rho_{1}+\bar{C}^{T} \bar{C}+Q & P \bar{A}_{d} & P \bar{G} \\
\bar{A}_{d}^{T} P & -Q & 0 \\
\bar{G}^{T} P & 0 & -\varepsilon I
\end{array}\right]<0
$$

which implies that if $w(t)=0$, then $\dot{V}_{2}<0$. If (35) holds, then (46) holds. Hence, system (8) is stable.

The proof is completed.

The last step of fault detection is to evaluate the residual. This is a decision making process that always comes down to a threshold logic of a decision function. From Assumption 2 $\left(d \in L_{2}\right)$, we can further have $\|d(t)\|_{2}=S$, where $S>0$ is a constant. By using Theorem 9, we obtain $\gamma_{d}=\sup _{d \in L_{2}}\|r\|_{2} /\|d\|_{2}$. In the fault-free case, the generated residual $r(t)$ is only affected by the disturbance input $d(t)$. Therefore, the threshold $J_{\text {th }}$ can be determined by

$$
J_{\mathrm{th}}=\gamma_{d}\|d(t)\|_{2}=\gamma_{d} S \text {. }
$$

The fault detection decision logic can be designed as

$$
\begin{aligned}
& \|r(t)\|_{2, \tau}>J_{\text {th }} \Longrightarrow \text { a fault has occured } \Longrightarrow \text { alarm } \\
& \|r(t)\|_{2, \tau}<J_{\text {th }} \Longrightarrow \text { no fault has occured. }
\end{aligned}
$$

Remark 10. In [18], $\Psi$ was described as $\Psi=g(x(t), x(t-h))-$ $g(\widehat{x}(t), \widehat{x}(t-h))$. From Assumption 2 in [18], one has

$$
\begin{aligned}
\|\Psi\| \leq & \left\|\rho_{1}(x(t)-\widehat{x}(t))\right\| \\
& +\left\|\rho_{2}(x(t-h)-\widehat{x}(t-h))\right\| \\
\leq & \left\|\rho_{1} e(t)\right\|+\left\|\rho_{2} e(t-h)\right\|, \\
\|\Psi\|^{2} \leq & 2\left\|\rho_{1} e(t)\right\|^{2}+2\left\|\rho_{2} e(t-h)\right\|^{2} .
\end{aligned}
$$

However, if time-delay $h$ is an unknown constant, $\|\Psi\|$ and $\|\Psi\|^{2}$ are not available and can not be used in the design procedure. In this paper, an improved observer is proposed, in which $h$ is replaced by the estimation value $\widehat{h}$. Then, $\Psi$ is presented in the following form:

$$
\Psi=g(x(t), x(t-h))-g(\widehat{x}(t), \widehat{x}(t-\widehat{h})) .
$$

Furthermore, by using Newton-Leibniz Formula, one has

$$
\begin{aligned}
\|\Psi\| \leq & \left\|\rho_{1}(x(t)-\hat{x}(t))\right\| \\
& +\left\|\rho_{2}(x(t-h)-\hat{x}(t-\widehat{h}))\right\| \\
\leq & \left\|\rho_{1} e(t)\right\|+\left\|\rho_{2} \int_{-\hat{h}}^{-h} \dot{\hat{x}}(t+s) d s\right\| .
\end{aligned}
$$

3.3. Fault Estimation. After detecting the fault, fault isolation and estimation are considered. Similar to [21], by a bank of so-called fault isolation observers, fault pattern and location can be obtained. In this paper, it is assumed that faults have been isolated and fault isolation is not studied further. In the following, we will investigate the fault estimation problem.

In order to estimate the fault, a following observer is constructed:

$$
\begin{aligned}
\dot{\hat{x}}(t)= & A \widehat{x}(t)+A_{d} \widehat{x}(t-\widehat{h})+B u(t) \\
& +G g(\widehat{x}(t), \widehat{x}(t-\widehat{h}))+B_{f} \widehat{f}(t) \\
& +H[y(t)-\widehat{y}(t)], \\
\hat{y}(t)= & C \widehat{x}(t)+D u(t)+D_{f} \widehat{f}(t), \\
r(t)= & V[y(t)-\widehat{y}(t)],
\end{aligned}
$$


where $\widehat{x}(t) \in R^{n}$ is the observer state vector, $\widehat{y}(t) \in R^{q}$ is the observer output vector, $\widehat{h}$ and $\widehat{f}$ are the estimation values of $h$ and $f$, respectively, and $r(t)$ is the so-called generated residual signal. The observer gain matrix $H$ and the residual weighting matrix $V$ will be defined later.

Let

$$
\begin{aligned}
& e=x(t)-\widehat{x}(t), \\
& \tilde{f}=f(t)-\widehat{f}(t) .
\end{aligned}
$$

Then, the error dynamics can be described as

$$
\begin{aligned}
\dot{e}(t)= & (A-H C) e(t)+A_{d} \widehat{x}(t-\widehat{h})-A_{d} x(t-h) \\
& +\left(B_{f}-H D_{f}\right) \tilde{f}(t)+\left(B_{d}-H D_{d}\right) d(t) \\
& +G \Psi \\
= & (A-H C) e(t)+A_{d} \widehat{x}(t-\widehat{h})-A_{d} \widehat{x}(t-h) \\
& +A_{d} \widehat{x}(t-h)-A_{d} x(t-h) \\
& +\left(B_{f}-H D_{f}\right) \tilde{f}(t)+\left(B_{d}-H D_{d}\right) d(t) \\
& +G \Psi \\
= & (A-H C) e(t)+A_{d} e(t-h)+A_{d} \widehat{x}(t-\widehat{h})
\end{aligned}
$$

$$
\begin{aligned}
& -A_{d} \widehat{x}(t-h)+\left(B_{f}-H D_{f}\right) \tilde{f}(t) \\
& +\left(B_{d}-H D_{d}\right) d(t)+G \Psi
\end{aligned}
$$

where $\Psi=g(x(t), x(t-h))-g(\widehat{x}(t), \widehat{x}(t-\widehat{h}))$.

By using Newton-Leibniz Formula, one has

$$
\widehat{x}(t-\widehat{h})-\widehat{x}(t-h)=\int_{-h}^{-\widehat{h}} \dot{\hat{x}}(t+s) d s .
$$

Equation (54) can be rewritten as

$$
\begin{aligned}
\dot{e}(t)= & (A-H C) e(t)+A_{d} e(t-h) \\
& +A_{d} \int_{-h}^{-\widehat{h}} \dot{\hat{x}}(t+s) d s+\left(B_{f}-H D_{f}\right) \tilde{f}(t) \\
& +\left(B_{d}-H D_{d}\right) d(t)+G \Psi, \\
r(t)= & V C e(t)+V D_{f} \tilde{f}(t)+V D_{d} d(t) .
\end{aligned}
$$

To realize the above-mentioned fault diagnosis objective, the following Theorem 11 is proposed.

Theorem 11. Consider system (1) subject to Assumptions 1-3. For a given positive constant $\alpha>0$, if there exist a scalar $\varepsilon>0$ and matrices $P_{1}=P_{1}^{T}>0, P_{2}=P_{2}^{T}>0, Q_{1}=Q_{1}^{T}>0$, $Q_{2}=Q_{2}^{T}>0, Y$, and $V$ such that

$$
\begin{aligned}
\Xi & =\left[\begin{array}{cccccccc}
\Omega_{1} & 0 & P_{1} A_{d} & 0 & P_{1} B_{f}-Y D_{f} & P_{1} B_{d}-Y D_{d} & C^{T} V^{T} & P_{1} G \\
* & \Omega_{2} & 0 & P_{2} A_{d} & P_{2}\left(B_{f}-\bar{H} D_{f}\right) & P_{2}\left(B_{d}-\bar{H} D_{d}\right) & -C^{T} \bar{V}^{T} & 0 \\
* & * & 2 \varepsilon \rho_{2}^{T} \rho_{2}-Q_{1} & 0 & 0 & 0 & 0 & 0 \\
* & * & * & Q_{2} & 0 & 0 & 0 & 0 \\
* & * & * & * & -\gamma^{2} I & 0 & D_{f}^{T}\left(V^{T}-\bar{V}^{T}\right) & 0 \\
* & * & * & * & * & -\gamma^{2} I & D_{d}^{T}\left(V^{T}-\bar{V}^{T}\right) & 0 \\
* & * & * & * & * & * & -I & 0 \\
* & * & * & * & * & * & * & -\varepsilon I
\end{array}\right]<0, \\
F C & =\left(B_{f}-H D_{f}\right)^{T} P
\end{aligned}
$$

then, using the following adaptive laws:

$$
\begin{aligned}
\dot{\hat{h}} & =-\frac{1}{\eta} M\left(\left\|P A_{d}\right\|+\left\|P G \rho_{2}\right\|\right) \alpha(t), \quad \hat{h}(0)>\bar{h}, \\
\dot{\hat{f}}(t) & =-\eta_{f}^{-1} \Gamma F e_{y}(t), \quad \dot{\hat{f}}(0)=0,
\end{aligned}
$$

the closed-loop system (56) is stable with $\|e\|_{2}<\alpha\|d\|_{2}$, where $\alpha(t)=\|\dot{\hat{x}}(t)\|, \eta_{f}>0$, and $\Gamma=\Gamma^{T}>0$ are adaptive rates, and $\eta$ is selected such that $\widehat{h}(0) \geq \bar{h}, Q-I>0$, and I denotes the identity matrix with appropriate dimensions.
Proof. Define the following Lyapunov-Krasovskii function:

$$
\begin{aligned}
V_{3}(t) & =e^{T}(t) P e(t)+\int_{t-h}^{t} e^{T}(\tau) Q e(\tau) d \tau \\
+ & 2 M\left(\left\|P A_{d}\right\|+\left\|P G \rho_{2}\right\|\right) \int_{-\widehat{h}}^{-h} d \theta \int_{\theta}^{0} \alpha(t+s) d s \\
+ & \frac{\eta}{2}(\widehat{h}-h)^{2}+\eta_{f}(\widehat{f}-f)^{T} \Gamma^{-1}(\widehat{f}-f),
\end{aligned}
$$

where $P=\left[\begin{array}{cc}P_{1} & 0 \\ 0 & P_{2}\end{array}\right], Q=\left[\begin{array}{cc}Q_{1} & 0 \\ 0 & Q_{2}\end{array}\right]$. 
Differentiating $V_{3}$ with respect to time $t$, one has

$$
\begin{aligned}
\dot{V}_{3} \leq & e^{T}(t)\left(P(A-H C)+(A-H C)^{T} P\right) e(t) \\
& +e^{T}(t) P A_{d} A_{d}^{T} P e(t)+e^{T}(t-h) e(t-h)+2 e^{T}(t) \\
& \cdot P\left(A_{d} \int_{-h}^{-\widehat{h}} \hat{\hat{x}}(t+s) d s+G \Psi\right)+2 e^{T}(t) P\left(B_{f}\right. \\
& \left.-H D_{f}\right) \tilde{f}+2 e^{T}(t) P\left(B_{d}-H D_{d}\right) d(t)+e^{T}(t) \\
& \cdot Q e(t)-e^{T}(t-h) Q e(t-h)+\eta(\widehat{h}-h) \dot{\hat{h}} \\
& -2 M\left(\left\|P A_{d}\right\|+\left\|P G \rho_{2}\right\|\right)[(\widehat{h}-h) \alpha(t) \\
& \left.-\int_{-\widehat{h}}^{-h} \alpha(t+\theta) d \theta+\dot{\hat{h}} \int_{-\widehat{h}}^{0} \alpha(t+s) d s\right] \\
& +2 \eta_{f} \tilde{f}^{T} \Gamma^{-1} \dot{\hat{f}} .
\end{aligned}
$$

From Assumption 2 and $\|\Psi\| \leq\left\|\rho_{1} e(t)\right\|+\left\|\rho_{2} \int_{-\widehat{h}}^{-h} \dot{\hat{x}}(t+s) d s\right\|$, it yields

$$
\begin{aligned}
2 e^{T}(t) P G \Psi \leq & \varepsilon^{-1} e^{T}(t) P G G^{T} P e(t) \\
& +2 \varepsilon e^{T}(t) \rho_{1}^{T} \rho_{1} e(t)+2\|e(t)\| \\
& \cdot\left\|P G \rho_{2}\right\| \int_{-\widehat{h}}^{-h} \alpha(t+s) d s .
\end{aligned}
$$

Because $\dot{\hat{h}}=-(1 / \eta) M\left(\left\|P A_{d}\right\|+\left\|P G \rho_{2}\right\|\right) \alpha(t) \leq 0$, we have $\dot{\hat{h}} \int_{-\widehat{h}}^{0} \alpha(t+s) d s \leq 0$. Notice that $Q-I>0$; then

$$
\begin{aligned}
\dot{V}_{3} & \leq e^{T}(t)\left(P(A-H C)+(A-H C)^{T} P+P A_{d} A_{d}^{T} P\right. \\
& \left.+Q+\varepsilon^{-1} P G G^{T} P+2 \varepsilon \rho_{1}^{T} \rho_{1}\right) e(t)+2 e^{T}(t) P\left(B_{f}\right.
\end{aligned}
$$

$$
\begin{aligned}
& \left.-H D_{f}\right) \tilde{f}+2 e^{T}(t) P\left(B_{d}-H D_{d}\right) d(t) \\
& +2 \eta_{f} \widetilde{f}^{T} \Gamma^{-1} \dot{\hat{f}} \leq e^{T}(t)(P(A-H C) \\
& +(A-H C)^{T} P+P A_{d} A_{d}^{T} P+Q+\varepsilon^{-1} P G G^{T} P \\
& \left.+2 \varepsilon \rho_{1}^{T} \rho_{1}\right) e(t)-2 \widetilde{f}^{T} F D_{f} \tilde{f}(t)-2 \widetilde{f}^{T} F D_{d} d(t) \\
& +2 e^{T}(t) P\left(B_{d}-H D_{d}\right) d(t)
\end{aligned}
$$

Now, define the following performance index:

$$
\begin{aligned}
J_{3}= & \int_{0}^{\infty} e^{T}(t) e(t) d t-\alpha^{2} \int_{0}^{\infty} d^{T}(t) d(t) d t \\
= & \int_{0}^{\infty}\left[e^{T}(t) e(t)-\alpha^{2} d^{T}(t) d(t)+\dot{V}_{3}(t)\right] d t \\
& -\left.V_{1}(t)\right|_{t}+\left.V_{3}(t)\right|_{t=0}
\end{aligned}
$$

Since under zero initial condition and $\widehat{h}(0)>h$ and $\left.V_{1}(t)\right|_{t=0}=0, V_{1}(t) \geq 0$ for all $t$. Thus,

$$
\begin{aligned}
J_{3} & <\int_{0}^{\infty}\left[e ^ { T } ( t ) \left(P(A-H C)+(A-H C)^{T} P\right.\right. \\
& \left.+P A_{d} A_{d}^{T} P+Q+\varepsilon^{-1} P G G^{T} P+2 \varepsilon \rho_{1}^{T} \rho_{1}+I\right) e(t) \\
& -2 \tilde{f}^{T} F D_{f} \tilde{f}(t)-2 \tilde{f}^{T} F D_{d} d(t)+2 e^{T}(t) P\left(B_{d}\right. \\
& \left.\left.-H D_{d}\right) d(t)-\alpha^{2} d^{T}(t) d(t)\right] d t
\end{aligned}
$$

which can be rewritten as

$$
J_{3}<\int_{0}^{\infty}\left[\begin{array}{c}
e(t) \\
\tilde{f}(t) \\
d(t)
\end{array}\right]^{T} \Xi_{1}\left[\begin{array}{l}
e(t) \\
\tilde{f}(t) \\
d(t)
\end{array}\right] d t
$$

where

$$
\Xi_{1}=\left[\begin{array}{ccc}
P(A-H C)+(A-H C)^{T} P+P A_{d} A_{d}^{T} P+Q+\varepsilon^{-1} P G G^{T} P+2 \varepsilon \rho_{1}^{T} \rho_{1}+I & 0 & \left(B_{d}-H D_{d}\right)^{T} P \\
0 & -F D_{f} & -D_{d}^{T} F \\
P\left(B_{d}-H D_{d}\right) & -D_{d} F & -\alpha^{2} I
\end{array}\right]
$$

From (57), one has $\Xi_{1}<0$. Therefore, $J_{3}<0$ with $\|e\|_{2}<$ $\alpha\|d\|_{2}$. If

$$
\Xi_{1}^{\prime}=\left[\begin{array}{ccc}
P(A-H C)+(A-H C)^{T} P+P A_{d} A_{d}^{T} P+Q+\varepsilon^{-1} P G G^{T} P+2 \varepsilon \rho_{1}^{T} \rho_{1}+I & 0 & 0 \\
0 & -F D_{f} & 0 \\
0 & 0 & -\alpha^{2} I
\end{array}\right]<0
$$


holds, then, $\dot{V}_{3}<0$ under the condition $d(t)=0$. Obviously, if (57) holds, then (68) holds. Therefore, system (56) is asymptotically stable.

The proof is completed.

Remark 12. Because of the existence of unknown disturbances, from the fault detection mechanism (54), the adaptive laws can be redesigned as

$$
\dot{\hat{f}}=P\left\{-\eta_{f}^{-1} \Gamma F e_{y}(t) D\left[e_{y}(t), J(t)\right]\right\},
$$

where the dead-zone operator $D[\cdot]$ is defined by

$$
D\left[e_{y}(t), r(t)\right]= \begin{cases}0, & \|J(t)\| \leq J_{\mathrm{th}} \\ e_{y}(t), & \|J(t)\|>J_{\mathrm{th}},\end{cases}
$$

where $J_{\text {th }}=\gamma_{d}\|d(t)\|_{2}=\gamma_{d} S$.

Remark 13. If there exist two known constants $f_{\min }, f_{\max }$ such that $f_{\min } \leq|f(t)| \leq f_{\max }$, then the fault $f(t)$ can be modeled in the following form:

$$
f(t)=\frac{1}{2}\left(f_{\max }-f_{\min }\right)(1-\tanh \theta)+f_{\min },
$$

where $\theta$ is an unknown constant. Thus, estimating the fault $f(t)$ consists in estimating $\theta$; that is to say

$$
\dot{\hat{f}}(t)=\frac{1}{2}\left(f_{\max }-f_{\min }\right)(1-\tanh \dot{\hat{\theta}})+f_{\text {min }}
$$

which prevents the phenomenon of parameter drift in the presence of bounded disturbances and ensures $f_{\min } \leq$ $|\widehat{f}(t)| \leq f_{\max }$.

\section{Simulation Results}

Consider the following time-delayed nonlinear system:

$$
\begin{aligned}
\dot{x}(t)= & A x(t)+A_{d} x(t-d)+B u(t) \\
& +G g(x(t), x(t-h))+B_{f} f(t)+B_{d} d(t), \\
y(t)= & C x(t)+D u(t)+G g(x(t), x(t-h)) \\
& +D_{f} f(t)+D_{d} d(t), \\
x(t)= & 0, \quad t \in[-h, 0],
\end{aligned}
$$

where

$$
\begin{aligned}
& A=\left[\begin{array}{cc}
-1.2 & 0.1 \\
-0.1 & -1.0
\end{array}\right], \\
& A_{d}=\left[\begin{array}{cc}
-0.6 & 0.7 \\
-1.0 & -0.8
\end{array}\right] \text {, } \\
& B=\left[\begin{array}{c}
0 \\
0.2
\end{array}\right] \text {, } \\
& C=\left[\begin{array}{ll}
1 & 0 \\
0 & 1
\end{array}\right], \\
& D=0, \\
& G=\left[\begin{array}{ll}
0.1 & 0.3 \\
0.2 & 0.5
\end{array}\right], \\
& B_{f}=\left[\begin{array}{cc}
0.7 & 0 \\
-0.5 & 0
\end{array}\right], \\
& B_{d}=\left[\begin{array}{ll}
0 & 1.0 \\
0 & 0.2
\end{array}\right], \\
& D_{f}=\left[\begin{array}{cc}
0 & -0.4 \\
0 & 0.8
\end{array}\right], \\
& D_{d}=\left[\begin{array}{ll}
1.5 & 0 \\
0.2 & 0
\end{array}\right], \\
& g(x(t), x(t-h))=\sin 0.2 x(t)+\sin 0.1 x(t-h), \\
& h=0.5 \mathrm{~s}, \\
& d=0.1 \sin t
\end{aligned}
$$

From Theorem 6, by solving LMI (19), one has

$$
\begin{aligned}
P & =\left[\begin{array}{ll}
0.6248 & 0.0610 \\
0.0610 & 0.4262
\end{array}\right], \\
Q & =\left[\begin{array}{cc}
1.1752 & -0.1434 \\
-0.1434 & 1.4028
\end{array}\right], \\
Z & =\left[\begin{array}{ll}
0.8685 & 0.0672 \\
0.0672 & 1.8887
\end{array}\right], \\
Y & =\left[\begin{array}{ll}
0.8536 & -0.5914 \\
0.2573 & 1.9270
\end{array}\right], \\
\alpha_{\min } & =0.6391 .
\end{aligned}
$$


Furthermore, we obtain the observer gain matrix $\bar{H}$ and the residual weighting matrix $\bar{V}$,

$$
\begin{aligned}
& \bar{H}=\left[\begin{array}{cc}
1.3259 & -1.4076 \\
0.4140 & 4.7225
\end{array}\right], \\
& \bar{V}=\left[\begin{array}{ll}
0.9315 & 0.0291 \\
0.0291 & 1.3740
\end{array}\right] .
\end{aligned}
$$

In this study, $\varepsilon, \rho_{1}, \rho_{2}$ are selected as $[16,18]$

$$
\begin{aligned}
\varepsilon & =0.1, \\
\rho_{1} & =\left[\begin{array}{ll}
0.2 & 0.1 \\
0.3 & 0.2
\end{array}\right], \\
\rho_{2} & =\left[\begin{array}{ll}
0.1 & 0.2 \\
0.3 & 0.1
\end{array}\right] .
\end{aligned}
$$

By using Theorem 11, we obtain the solutions of LMI (35) with $P_{1}, P_{2}, Q_{1}, Q_{2}, V, Y$ and minimal $\gamma_{\text {min }}$, respectively, as follows:

$$
\begin{aligned}
P_{1} & =\left[\begin{array}{rr}
24.0724 & -12.6296 \\
-12.6296 & 10.7939
\end{array}\right], \\
Q_{1} & =\left[\begin{array}{ll}
44.0436 & 2.0634 \\
2.0634 & 71.8486
\end{array}\right], \\
P_{2} & =\left[\begin{array}{ll}
29.9378 & 8.3931 \\
8.3931 & 16.0890
\end{array}\right], \\
Q_{2} & =\left[\begin{array}{ll}
64.3589 & 17.5958 \\
17.5958 & 67.3153
\end{array}\right], \\
V & =\left[\begin{array}{ll}
0.4468 & -0.0364 \\
-0.0364 & 0.0128
\end{array}\right], \\
Y & =\left[\begin{array}{ll}
35.0527 & 28.7520 \\
28.7520 & 78.3480
\end{array}\right], \\
\gamma_{\min } & =0.6402 .
\end{aligned}
$$

Then, one has the observer gain matrix $H$ :

$$
H=\left[\begin{array}{ll}
4.2442 & 12.9558 \\
5.3140 & 22.4176
\end{array}\right]
$$

In this paper, to illustrate the effectiveness of the proposed algorithm, two fault cases are considered: abrupt fault case and incipient fault case.

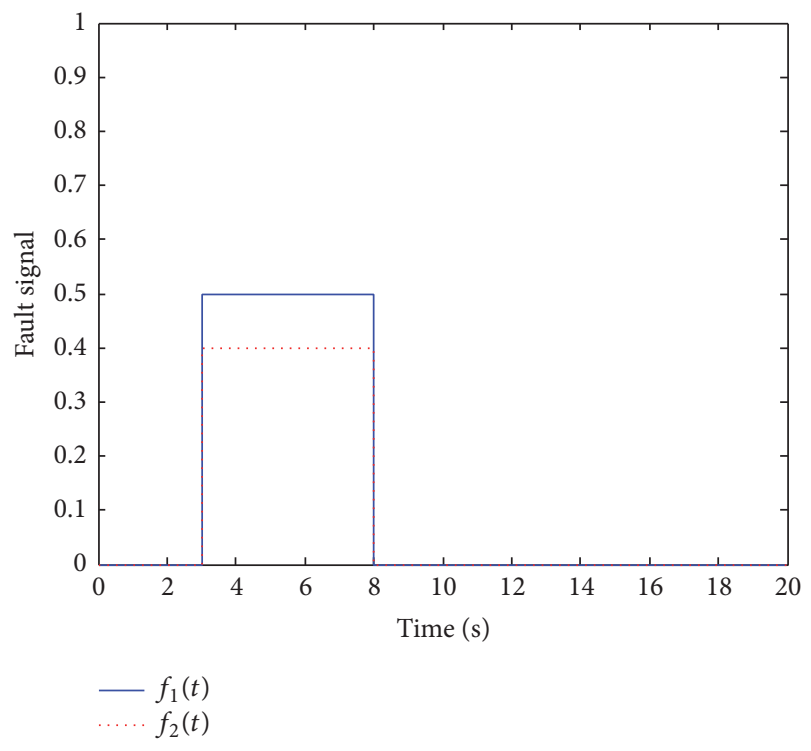

FIGURE 1: Fault signal $f(t)$.

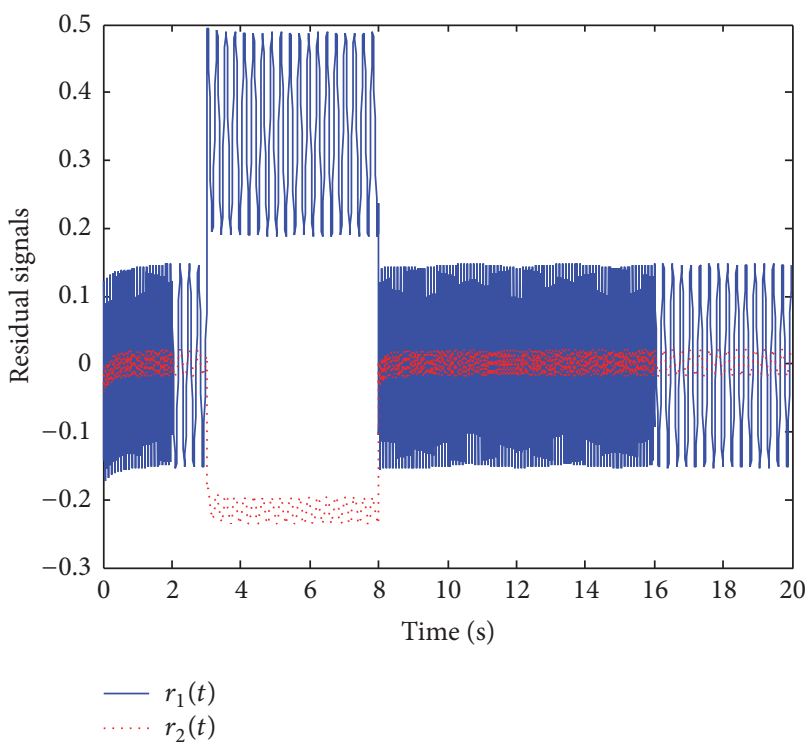

Figure 2: Generated residual $r(t)$.

4.1. Abrupt Fault Case. In this case, fault $f(t)=\left[f_{1}(t), f_{2}(t)\right]^{T}$ is defined in following form:

$$
f_{1}(t)= \begin{cases}0, & 0 \leq t \leq 3 \\ 0.5, & 3<t \leq 8 \\ 0, & t>8\end{cases}
$$$$
f_{2}(t)= \begin{cases}0, & 0 \leq t \leq 3 \\ 0.4, & 3<t \leq 8 \\ 0, & t>8 .\end{cases}
$$

The fault signal $f(t)$ and the generated residual signals $r(t)$ (including $r_{1}(t)$ and $\left.r_{2}(t)\right)$ are shown in Figures 1 and 2 , respectively. Figure 3 shows the evolution of residual 


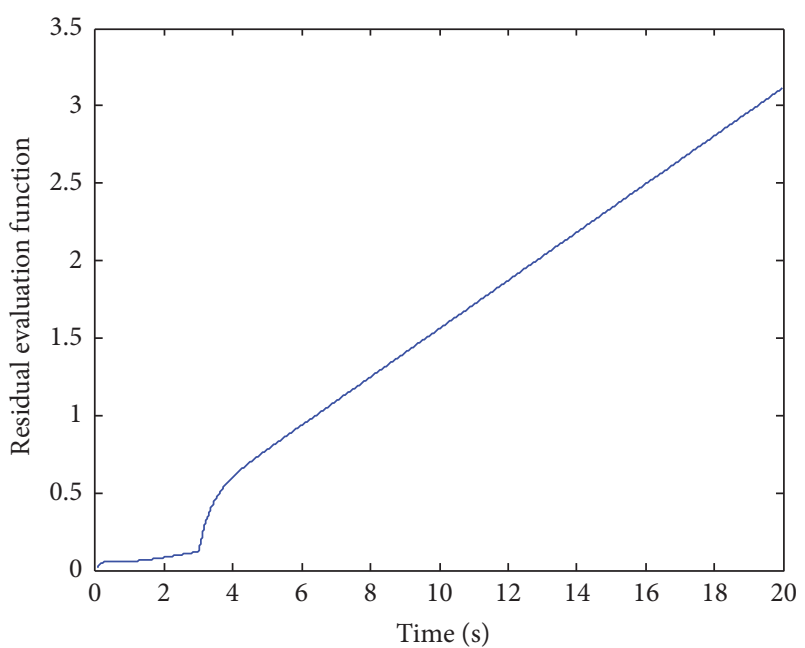

Figure 3: Evolution of the residual evaluation function $\|r(t)\|$.

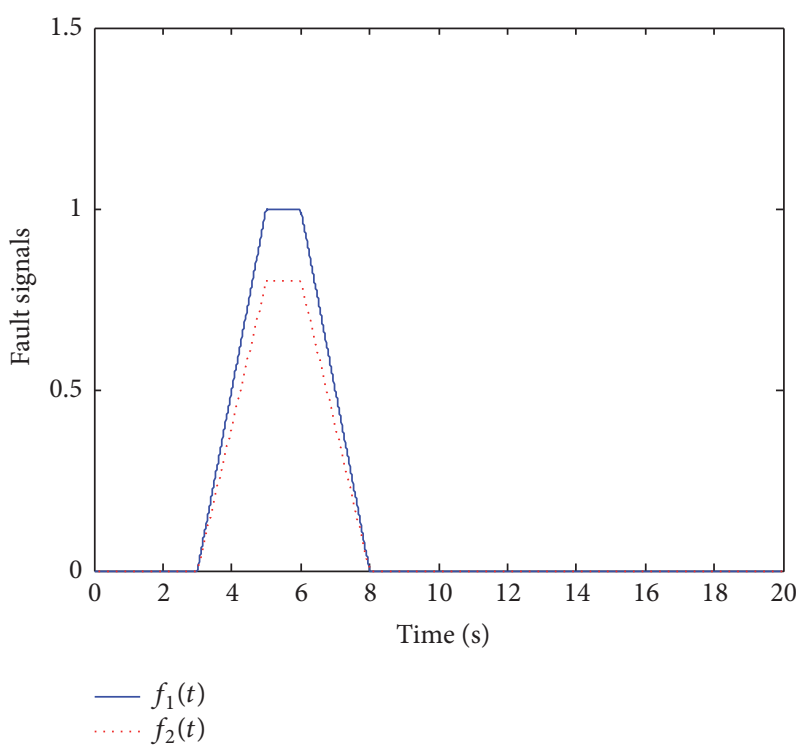

Figure 4: Fault signal $f(t)$.

evaluation function $\|r(t)\|$, from which the fault can be detected.

4.2. Incipient Fault Case. As pointed out in [18], in many real physical systems, the fault evolves slowly. In this case, the fault $f(t)$ is assumed to be an incipient fault, which is shown in Figure 4. The generated residual signals $r(t)$ (including $r_{1}(t)$ and $\left.r_{2}(t)\right)$ and the evolution of residual evaluation function are illustrated in Figures 5 and 6, respectively.

\section{Conclusions}

In this paper, the problem of fault detection and estimation of nonlinear time-delayed systems with constant but unknown state time-delay is studied. A new fault detection filter with
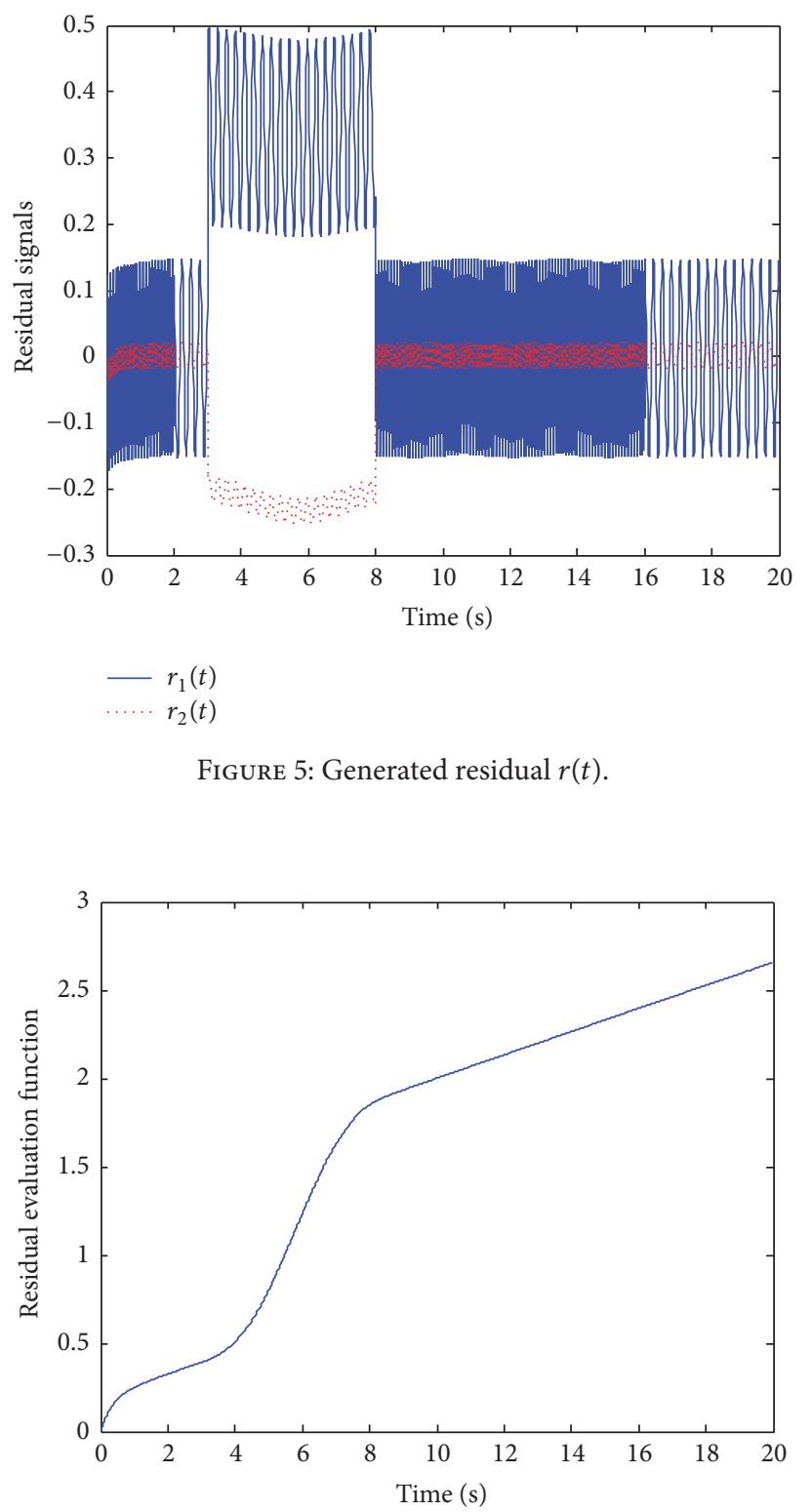

Figure 6: Evolution of the residual evaluation function $\|r(t)\|$.

adaptation to the time-delay is proposed. Then, a reference residual model is introduced to formulate the robust fault detection filter design problem as an $H_{\infty}$ model-matching problem. A novel robust adaptive fault estimation algorithm is proposed where the time derivative of the output errors has not been computed. In addition, applying a robust $H_{\infty}$ optimization control technique, sufficient conditions for the existence of the fault detection filter are derived in terms of LMI. In future, we will consider the fault diagnosis and fault tolerant control of actuators and sensors when both the input and the state are time-delay.

\section{Conflicts of Interest}

The authors declare that they have no conflicts of interest. 


\section{Acknowledgments}

This work is supported by National Natural Science Foundation of China (61503156, 61403161, and 61473250) and the Fundamental Research Funds for the Central Universities (JUSRP11562, NJ20150011) and National Key Research and Development Program (2016YFD0400301).

\section{References}

[1] J. Liu, W. Luo, X. Yang, and L. Wu, "Robust Model-Based Fault Diagnosis for PEM Fuel Cell Air-Feed System," IEEE Transactions on Industrial Electronics, vol. 63, no. 5, pp. 32613270, 2016.

[2] J. Liu, C. Wu, Z. Wang, and L. Wu, "Reliable Filter Design for Sensor Networks in the Type-2 Fuzzy Framework," IEEE Transactions on Industrial Informatics, vol. PP, no. 99, 1 page, 2017.

[3] Q. Zhao and J. Jiang, "Reliable state feedback control systems designs against actuator failures," Automatica, vol. 34, no. 10, pp. 1267-1272, 1998.

[4] X. Tang, G. Tao, and S. M. Joshi, "Adaptive output feedback actuator failure compensation for a class of non-linear systems," International Journal of Adaptive Control and Signal Processing, vol. 19, no. 6, pp. 419-444, 2005.

[5] X.-G. Yan and C. Edwards, "Nonlinear robust fault reconstruction and estimation using a sliding mode observer," Automatica. A Journal of IFAC, the International Federation of Automatic Control, vol. 43, no. 9, pp. 1605-1614, 2007.

[6] B. Jiang, M. Staroswiecki, and V. Cocquempot, "Fault accommodation for nonlinear dynamic systems," Institute of Electrical and Electronics Engineers. Transactions on Automatic Control, vol. 51, no. 9, pp. 1578-1583, 2006.

[7] H. Yang, B. Jiang, and V. Cocquempot, Fault tolerant control design for hybrid systems, vol. 397 of Lecture Notes in Control and Information Sciences (LNCIS), Springer Verlag, 2010.

[8] H. Yang, B. Jiang, V. Cocquempot, and L. Lu, "Supervisory fault tolerant control with integrated fault detection and isolation: a switched system approach," International Journal of Applied Mathematics and Computer Science, vol. 22, no. 1, pp. 87-97, 2012.

[9] M. Blanke, M. Kinnaert, J. Lunze, and M. Staroswiecki, Diagnosis and fault-tolerant control, Springer, Heidelberg, Third edition, 2016.

[10] T. Steffen, Control Reconfiguration OfDynamical Systems: Linear Approaches And Structural Tests Lecture Notes in Control and Information Sciences (LNCIS), vol. 320 of Lecture Notes in Control and Information Sciences, Springer, Berlin, Germany, 2005.

[11] J.-P. Richard, "Time-delay systems: an overview of some recent advances and open problems," Automatica. A Journal of IFAC, the International Federation of Automatic Control, vol. 39, no. 10, pp. 1667-1694, 2003.

[12] X.-G. Yan, S. K. Spurgeon, and C. Edwards, "Decentralised stabilisation for nonlinear time delay interconnected systems using static output feedback," Automatica. A Journal of IFAC, the International Federation of Automatic Control, vol. 49, no. 2, pp. 633-641, 2013.

[13] B. Jiang, J. L. Wang, and Y. C. Soh, "An adaptive technique for robust diagnosis of faults with independent effects on system outputs," International Journal of Control, vol. 75, no. 11, pp. 792802, 2002.
[14] X. Jiang, W. Xu, and Q.-L. Han, "Observer-based fuzzy control design with adaptation to delay parameter for time-delay systems," Fuzzy Sets and Systems. An International Journal in Information Science and Engineering, vol. 152, no. 3, pp. 637-649, 2005.

[15] C. Jiang and D. H. Zhou, "Fault detection and identification for uncertain linear time-delay systems," Computers and Chemical Engineering, vol. 30, no. 2, pp. 228-242, 2005.

[16] J. Kim and M. K. P, "Fault detection for time-delay system by using robust control theory," in Proceedings of the ISIE, pp. 869873, 2001.

[17] S. X. Ding, Z. Maiying, T. Bingyong, and P. Zhang, "An LMI approach to the design of fault detection filter for time-delay LTI systems with unknown input," in Proceedings of the 2001 American Control Conference, pp. 2137-2142, usa, June 2001.

[18] L. Bai, Z. Tian, and S. Shi, "Robust fault detection for a class of nonlinear time-delay systems," Journal of the Franklin Institute. Engineering and Applied Mathematics, vol. 344, no. 6, pp. 873888, 2007.

[19] B. Jiang, M. Staroswiecki, and V. Cocquempot, "Ho fault detection filter design for linear discrete-time systems with multiple time-delays," International Journal of Systems Science, vol. 34, no. 5, pp. 365-373, 2003.

[20] M. Zhong, S. X. Ding, J. Lam, and C. Zhang, "Fault Detection Filter Design for LTI System with Time Delays," in Proceedings of the 42nd IEEE Conference on Decision and Control, pp. 14671472, 2003.

[21] B. Jiang, M. Staroswiecki, and V. Cocquempot, "Fault identification for a class of time-delay systems," in Proceedings of the American Control Conference, pp. 2239-2244, Anchorage, Alaska, USA, 2002.

[22] K. Zhang and B. Jiang, "Analysis and design of adaptive fault estimation for time-varying delay systems," in Proceedings of the 27th Chinese Control Conference, CCC, pp. 38-42, chn, July 2008.

[23] S. Vazquez, . Jianxing Liu, . Huijun Gao, and L. G. Franquelo, "Extended State Observer-Based Sliding-Mode Control for Three-Phase Power Converters," in Proceedings of the IEEE Transactions on Industrial Electronics, vol. 64, pp. 22-31, Yokohama, 2016.

[24] P. D. Roberts, Robust Model-based Fault Diagnosis for Dynamic Systems, vol. 11, Kluwer Academic Publishers, Boston, Mass, USA, 2001.

[25] M. Zhong, S. X. Ding, J. Lam, and H. Wang, "An LMI approach to design robust fault detection filter for uncertain LTI systems," Automatica. A Journal of IFAC, the International Federation of Automatic Control, vol. 39, no. 3, pp. 543-550, 2003. 


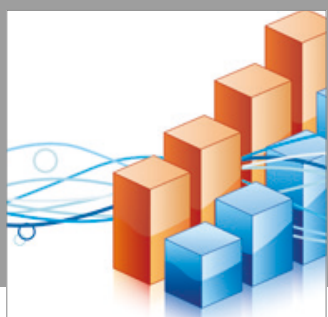

Advances in

Operations Research

vatersals

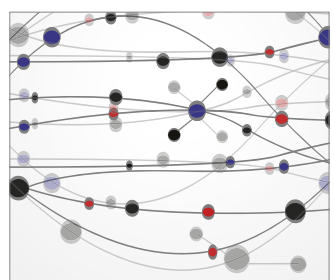

\section{The Scientific} World Journal
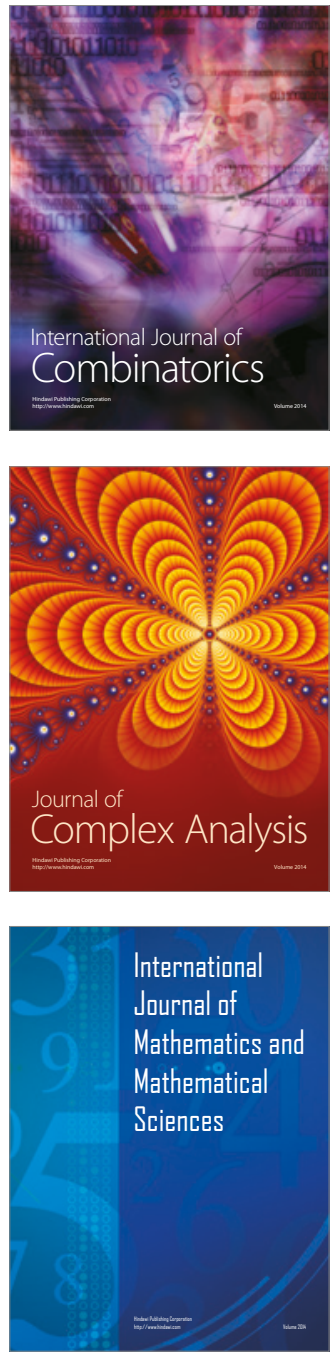
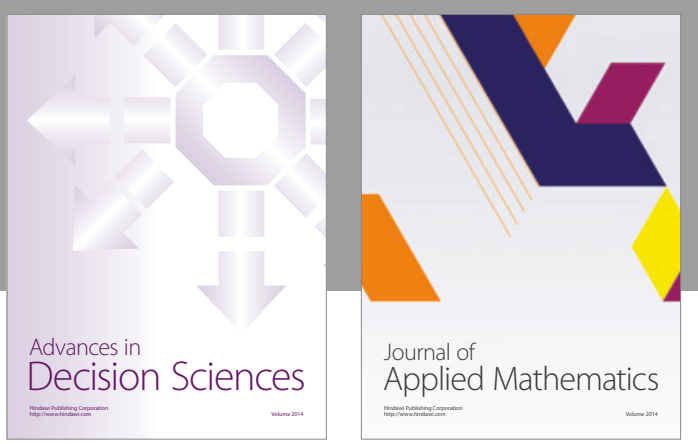

Algebra

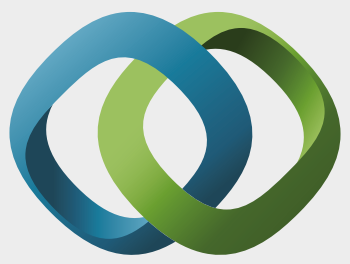

\section{Hindawi}

Submit your manuscripts at

https://www.hindawi.com
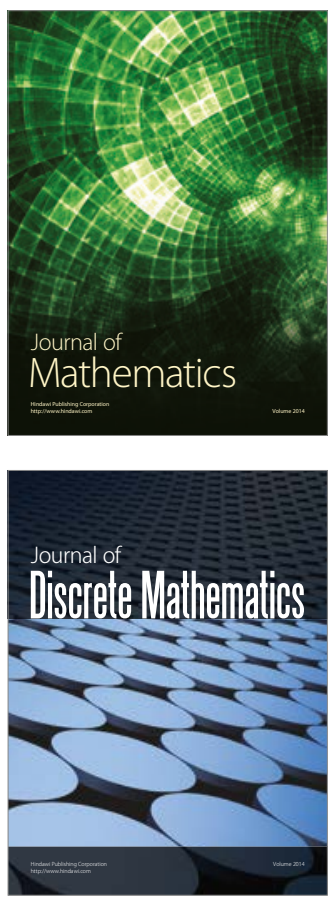

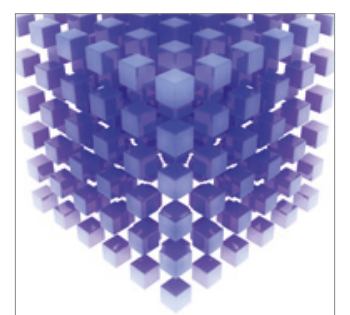

Mathematical Problems in Engineering
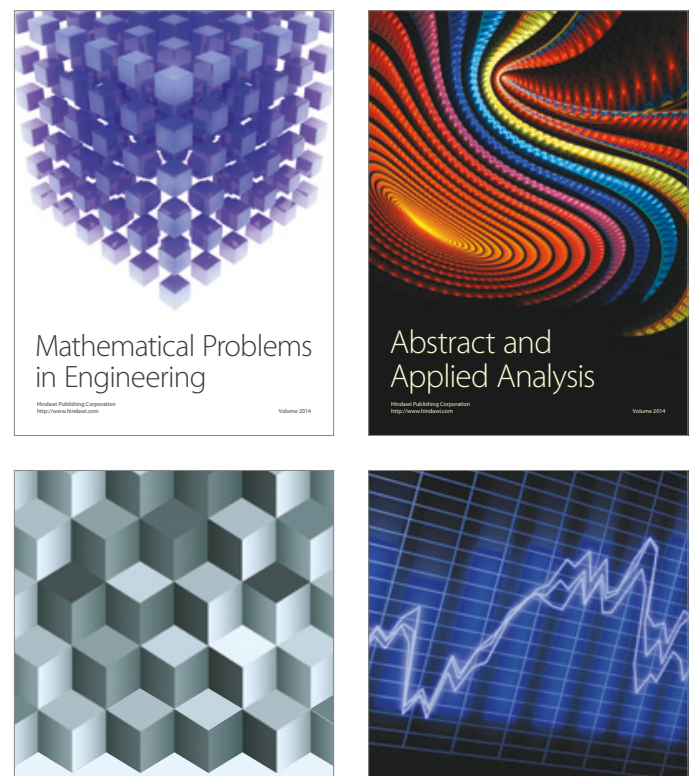

Journal of

Function Spaces

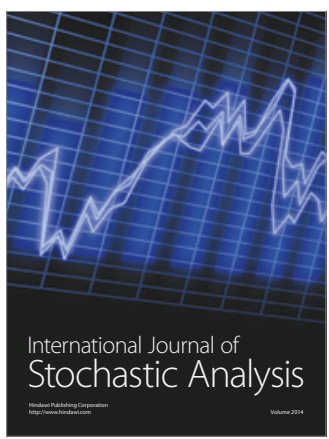

Probability and Statistics
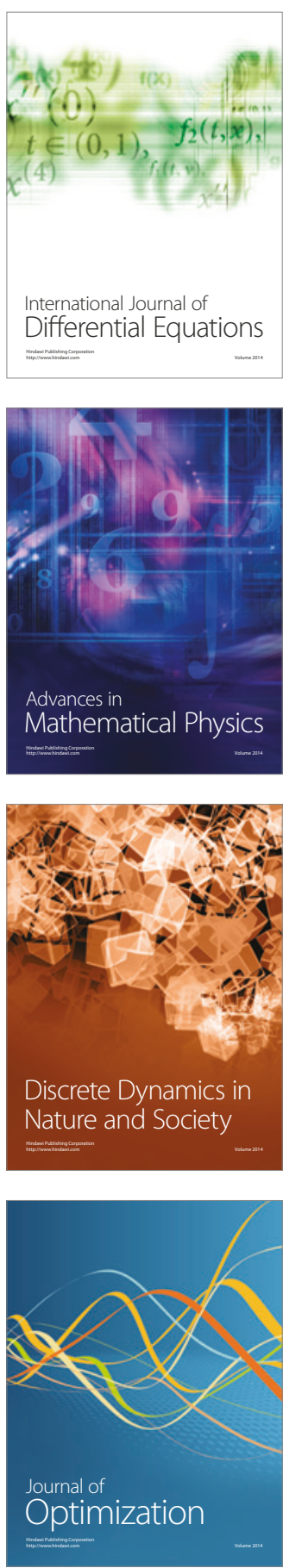